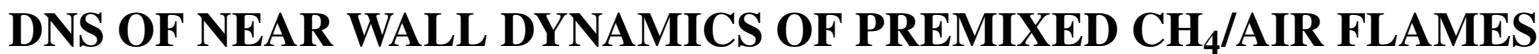

\section{Feichi Zhang ${ }^{1, *}$, Thorsten Zirwes ${ }^{2,1}$, Thomas Häber ${ }^{3}$, Henning Bockhorn ${ }^{1}$, Dimosthenis Trimis ${ }^{1}$ and Rainer Suntz ${ }^{3}$}

${ }^{1}$ Engler-Bunte-Institute, Division of Combustion Technology, Karlsruhe Institute of Technology Engler-Bunte-Ring 1, 76131 Karlsruhe, Germany

E-mail feichi.zhang@kit.edu; henning.bockhorn@kit.edu; dimosthenis.trimis@kit.edu

${ }^{2}$ Steinbuch Centre for Computing (SCC), SimLab Energy \& Competence Centre ING, Karlsruhe Institute of Technology

Hermann-von-Helmholtz-Platz 1, Karlsruhe, Germany

thorsten.zirwes@kit.edu

${ }^{3}$ Institute of Chemical Technology and Polymer Chemistry, Karlsruhe Institute of Technology Engesserstr. 20, 76131 Karlsruhe, Germany

thomas.haeber@kit.edu; suntz.rainer@kit.edu

Key words: Flame-wall interactions (FWI); Side-wall quenching (SWQ); Flame Dynamics; Markstein effect; Direct numerical simulation; OpenFOAM

Abstract. This work presents a numerical study on the effect of flame-wall interaction (FWI) from the viewpoint of flame dynamics. For that purpose, direct numerical simulations (DNS) employing detailed calculations of reaction rates and transport coefficients have been applied to a 2D premixed methane/air flame under atmospheric condition. Free flame (FF) and side-wall quenching (SWQ) configurations are realized by defining one lateral boundary as either a symmetry plane for the FF or a cold wall with fixed temperature at $20{ }^{\circ} \mathrm{C}$ for the SWQ case. Different components of flame stretch and Markstein number regarding tangential, normal (due to curvature) and total stretch, $K a_{s}, K a_{c}$ and $K a_{t o t}=K a_{s}+K a_{c}$, as well as their correlations with respect to the local flame consumption speed $S_{L}$ have been evaluated.

It has been shown that the FWI zone is dominated by negative flame stretch. In addition, $S_{L}$ decreases with decreasing normal stretch due to curvature $K a_{c}$ while approaching the cold wall. However, $S_{L}$ increases with decreasing $K a_{c}$ while approaching the symmetry boundary for the free flame case, leading to an inversion of the Markstein number $M a_{t o t}$ based on $K a_{t o t}$ from positive in the free flame case to negative in the SWQ case. The quenching distance evaluated based on wall-normal profiles of $S_{L}$ has been found to be approximately equal to the unstretched laminar flame thickness, which compares quantitatively well with measured data from literature. The flame speed has been confirmed to scale quasi-linearly with the stretch in the FWI zone. The results reveal a distinct correlation during transition between FWI and FF regarding flame dynamics, which brings a new perspective for modeling FWI phenomena by means of flame stretch and Markstein number. To do this, the quenching effect of the wall may be reproduced by a reversed sign of the Markstein number from positive to negative in the FWI zone and by applying the general linear Markstein correlation $\left(S_{L} / S_{L, 0}=1-M a \cdot K a\right)$, leading to a decrease of the flame speed or the reaction rate in the near-wall region. 


\section{INTRODUCTION}

In order to achieve a high power density and to improve fuel efficiency, most combustion applications take place in an enclosed environment with a relatively small volume, for instance in internal combustion or jet engines. The flame in this case propagates against a cold wall and is extinguished in the direct vicinity of the wall. As a result, the phenomenon of flame-wall interaction (FWI) significantly contributes to the flame stability and efficiency of the combustion process, but also to the pollutant emissions and lifetime of the burner [1]. The effect of FWI has been studied extensively in the last years due to its importance [2, 3, 4, 5, 6, 7, 8, 9, 10]. For instance, Dreizler and Böhm [2] and Kosaka et al. [9] performed comprehensive laser diagnostics for detailed measurements of flow velocity, temperature, species concentrations and flame structure for premixed methane/air and DME/air flames in the FWI zone, which improved the understanding of the related chemo-physical phenomena substantially. The same experimental setup has then been studied numerically by Ganter et al. [5] and Heinrich et al. [4] using different reaction-diffusion models and 2D/3D simulations, where the calculated wallnormal temperature profiles, heat fluxes and wall distances showed a good agreement with measured data. Saffman [11] measured the quenching distances for different hydrocarbon fuels and equivalence ratios. The experimental and numerical works by Häber and Suntz [6] and Strassacker et al. [7] have been focused on the effect of different wall materials, including heterogeneous wall reactions. They found that the quenching distance is only weakly dependent on the wall material and type of coating. The 3D direct numerical simulations (DNS) performed by Gruber et al. [12] for turbulent hydrogen/air flame interacting with a cold wall showed that the local flame thickness and propagation speed vary near the boundary layer, resuti at the wall. In addition, on the convective heat the influence of wall tem instability can be controlled or numerical simulation by $\mathrm{p}$
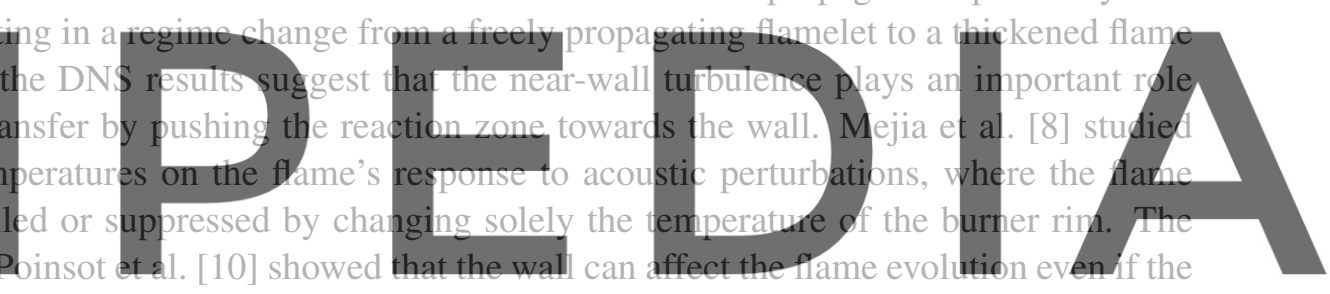

distance between the flame and the wall is significant. Popp and Baum [13] presented a numerical study

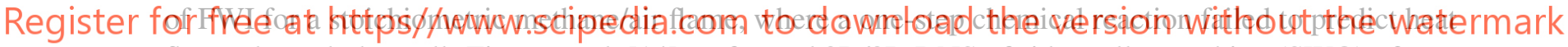
fluxes through the wall. Zirwes et al. [14] performed 2D/3D DNS of side-wall quenching (SWQ) of premixed methane/air flames at different equivalence ratios. The calculated quenching distances however showed a consistent constant deviation from the measured data.

Despite the progresses made in the previous works, studying FWI is challenging as the relevant phenomena occur in a very thin near-wall layer with a thickness of the order of $O(0.1 \mathrm{~mm})$. Focus of the recent numerical works was also to validate the proposed modeling concepts, such as the influences of using 2D/3D grids or detailed/reduced reaction-diffusion models, on reproducing the measured data $[4,5,7,15,14]$. In addition, mostly under-resolved grid resolutions have been applied in these works. Moreover, flames approaching walls are dominated by unsteady effects, which further complicate the FWI phenomena and make the comparison between measurement and simulation difficult. Therefore, more fine-grained simulations of FWI with sufficiently high grid resolutions, detailed reaction and transport models, as well as well-defined boundary conditions are necessary for a thorough understanding of the underlying processes of FWI. In an attempt to achieve this, DNS have been performed in the current work for a 2D side-wall quenching (SWQ) setup operated with premixed methane/air mixtures. Detailed chemical kinetics and molecular transport models are used and the FWI zone is resolved with a 
sufficiently fine grid. Special focus of the work is put on studying local flame dynamics regarding flame stretch, flame speed and the Markstein effect in the FWI zone, revealing systematically the significant role of flame dynamics for elucidating the mechanism of flame extinction at a cold wall. In comparison to our previous work shown in [16], the analysis of near wall flame dynamics has been further refined to identify different stages of flame dynamics during FWI.

\section{Theoretical Basics}

Flame stretch is calculated in this work by $[1,17]$ :

$$
K=(\mathbf{I}-\vec{n} \vec{n}): \nabla \vec{u}+S_{D} \nabla \cdot \vec{n}
$$

where $\mathbf{I}$ is the unit tensor, $\vec{u}$ the velocity vector and $\vec{n}$ the unit normal vector of the flame surface. The first term $K_{s}=(\mathbf{I}-\vec{n} \vec{n}): \nabla \vec{u}$ on the r.h.s. in Eq. (1) is attributed to hydrodynamic stretch caused by the non-uniform flow distribution and the second term $K_{c}=S_{d} \nabla \cdot \vec{n}$ due to the motion of a curved surface with the displacement speed $S_{D}$ along the flame normal direction. $\vec{n}=\frac{\nabla Y_{F}}{\left|\nabla Y_{F}\right|}$ and $S_{D}$ are evaluated at the flame surface defined by the fuel mass fraction $Y_{F}=Y_{F, \text { iso }}[18]$

$$
S_{D}=-\frac{1}{\left|\nabla Y_{F}\right|} \frac{\mathrm{d} Y_{F}}{\mathrm{~d} t}=-\frac{\dot{r}_{F}+\nabla \cdot\left(\rho D_{F} \nabla Y_{F}\right)}{\rho\left|\nabla Y_{F}\right|}
$$

$\dot{r}_{F}$ is the reaction rate of fuel, $\rho$ the density and $D_{F}$ the diffusion coefficient of fuel.

The laminar flame speed is evaluated in the present work by integration of the fuel reaction

local normals
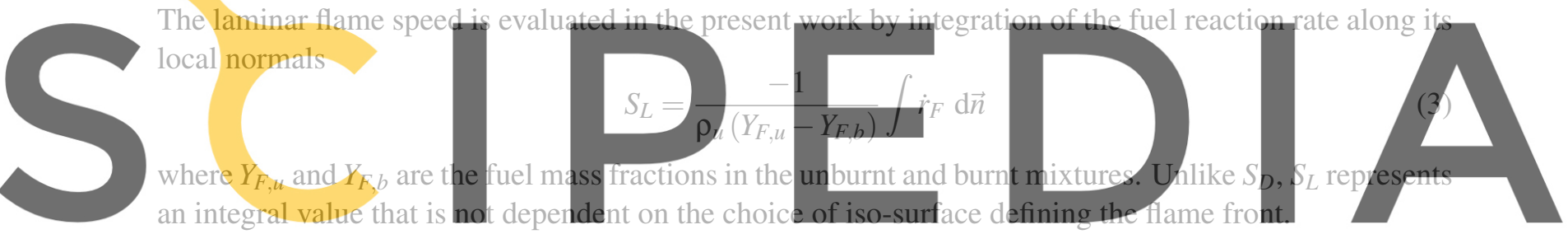

The influence of stretch on the laminar flame speed can be derived by means of a first order Taylor

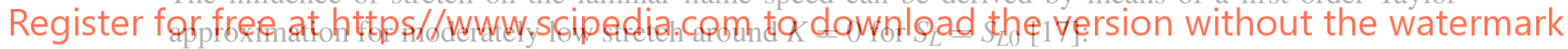

$$
S_{L}(K) \approx S_{L}(K=0)+\left.\frac{\partial S_{L}}{\partial K}\right|_{K=0}(K-0) \quad \text { or } \quad \frac{S_{L}(K)}{S_{L 0}}=1-\frac{\mathcal{L}}{S_{L 0}} K
$$

with the Markstein length $\mathcal{L}=-\partial S_{L} /\left.\partial K\right|_{K=0}$ and the unstretched laminar flame speed $S_{L 0}$. Equation (4) can be rewritten as

$$
\frac{S_{L}(K)}{S_{L 0}}=1-\frac{\mathcal{L}}{\delta_{L 0}} \frac{\delta_{L 0}}{S_{L 0}} K=1-M a K a
$$

to introduce the Markstein number $M a$ defined as the ratio of the Markstein length and the characteristic flame thickness $\delta_{L 0}$

$$
M a=\frac{\mathcal{L}}{\delta_{L 0}}=-\frac{1}{\delta_{L 0}} \frac{\partial S_{L}}{\partial K}
$$

and the Karlovitz number $K a=K \delta_{L 0} / S_{L 0}$. In this way, $M a$ measures the sensitivity of flame speed to stretch. A vast variety of experimental and computational work has been conducted in the last decades regarding the effect of stretch on flame speed $[19,20,21,22,23,24,18]$, which justified the asymptotic correlation proposed in Eq. (5). However, most of the previous studies have been conducted for 
1D canonical flame configurations with either outwardly expanding spherical flames or counterflow flames. The spherical flame is only positively curved and the opposed counterflow flame is only positively strained in tangential direction. In addition, the influence of heat loss on flame dynamics, for instance, due to a cold wall cannot be assessed. Therefore, detailed studies on flame dynamics are needed to extend existing knowledge for the combined impact of strain and curvature considering the negative stretch range, but also the effect of heat loss, for example, due to interaction with a cold wall.

\section{Numerical Setups}

Figure 1 shows a sketch of the 2D computational domain, which has a rectangular shape with lengths of $32 \mathrm{~mm}$ in streamwise and $8 \mathrm{~mm}$ in spanwise direction. The boundary conditions used for the four sides of the domain are indicated in Fig.1. Premixed methane/air mixture enters the domain at the inlet from the bottom with a temperature of $T_{0}=293 \mathrm{~K}$ and a pressure of $p_{0}=1$ bar. The gradients of flow velocity, temperature and all chemical scalars are set to zero at the outlet. A parabolic velocity profile is used for the inflow, with a minimum velocity of zero in the center and a maximum value of $\hat{u}=1.6 \mathrm{~m} / \mathrm{s}$ at both lateral ends. This leads to a V-shaped flame stabilized at the center and close to the inlet plane. The right side of the domain is set as a symmetry plane and the left boundary is defined either as a cold wall with $293 \mathrm{~K}$ or as a symmetry plane, as illustrated in Fig.1. These two simu-

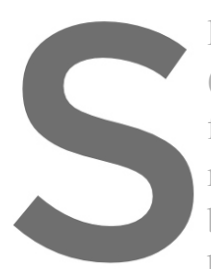
lation setups are identifiec (side-wall quenching) and flame branch propagate metry plane. In addition, been applied to the inlet boundaries. Premixed torethane/. flames at differcon
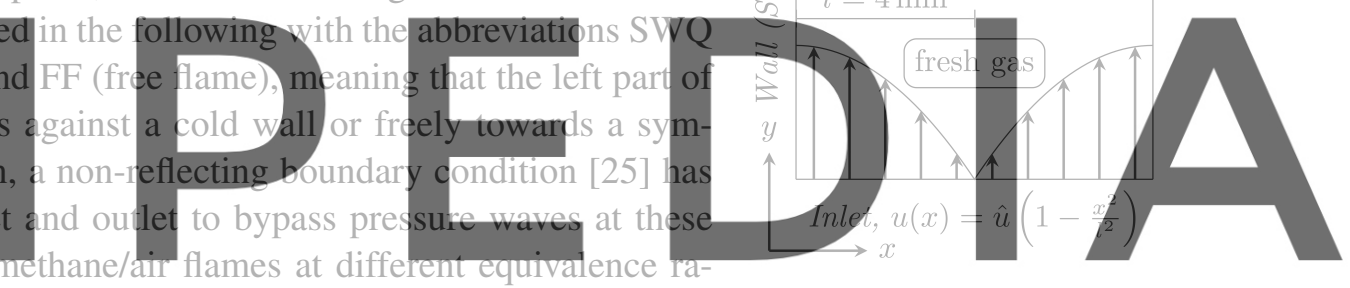
tios $\Phi=0.8,1.0$ and 1.2 are examined in this work. The laminar flame Figure 1: Computational domain and Register for free stretched freely propagating flames with Cantera. $S_{L}^{0}$ and $\delta_{L}^{0}$ from these simulations are used to evaluate $K a$ and Ma (see Eq.(5-6)). The filame surface is identified by the mass fraction of fuel corresponding to the highest consumption rate of fuel $Y_{\dot{r}_{\max }}$.

Table 1: Laminar flame parameters from 1D flame calculations at different $\Phi$ and $p_{0}=1 \mathrm{bar}, T_{0}=293 \mathrm{~K}$.

\begin{tabular}{c|ccc}
$\phi[-]$ & 0.8 & 1.0 & 1.2 \\
\hline$S_{L}^{0}[\mathrm{~cm} / \mathrm{s}]$ & 26.17 & 36.41 & 32.19 \\
$\delta_{L}^{0}[\mathrm{~mm}]$ & 0.536 & 0.445 & 0.472 \\
$Y_{\dot{r}_{\max }}[-]$ & 0.0039 & 0.0057 & 0.0068
\end{tabular}

The simulations have been performed using a DNS code developed in the framework of OpenFOAM, which includes detailed calculations of reaction rates and transport coefficients [26, 27, 28, 29, 30, 31, $32]$. For the DNS the conservation equations for total mass, momentum, species masses and energy are solved in the compressible formulations. The chemical reactions of the system are described by the GRI- 
3.0 mechanism [33] and molecular diffusion is considered with the mixture-averaged transport model. The simulations employ a fully implicit scheme of second order (backward) for the time derivative and a fourth order interpolation scheme for the discretization of the convective term. All diffusive terms are discretized with an unbounded scheme of fourth-order, too. The pressure-implicit split-operator (PISO) algorithm has been used for pressure correction. An equidistant grid length of $20 \mu \mathrm{m}$ is used for the simulations, which ensures resolving the unstretched laminar flame thickness with more than 20 cells. The time step has been set to $0.25 \mu \mathrm{s}$, corresponding to a maximum CFL number of 0.2.

\section{Results and Discussions}

Figure 2 from left to right shows the spatial distributions of the calculated streamwise velocity $v$, temperature $T$, mass fraction of methane $Y_{\mathrm{CH}_{4}}$ and consumption rate of methane $\dot{r}_{\mathrm{CH}_{4}}$ for the SWQ case with $\Phi=1$. The solid lines in Fig.2a indicate streamlines for visualization of the flow direction. The $\mathrm{V}$-shaped flame front can be identified by the large gradients of $T$ and $Y_{\mathrm{CH}_{4}}$ shown in Fig.2b and c. As the temperature at the inlet is fixed at the fresh gas temperature, the flame cannot stabilize directly at the inlet plane, which leads to a slightly lifted flame above the inlet. In Fig.2a, due to thermal expansion caused by the combustion reaction, the flow is accelerated by passing through the flame front, which leads to bending of the streamlines towards the flame-normal direction. The FWI zone can be identified from the contact point of the flame surface with the wall, where the flow velocity and reaction rate decrease to 0 , and the temperature decreases to the wall temperature. In this case, the flame is pushed away from the wall, which results in a temperature and velocity boundary layer downstream. The thickness of these boundary layers are smallest in the FWI zone
velocity are largest there.
A zoomed view of the FWI zone for the same cas
cated by the white line of $Y_{\mathrm{CH}_{4}}=Y_{\dot{r}_{\max }}$ (sce Tab.1).
grid resolution, where the reaction zone with $\dot{r}_{\mathrm{CH}_{4}}$ grid resolution, where the reaction zone with $\dot{r}_{\mathrm{CH}_{4}}$
near-wall region. The largest wall distance has bee

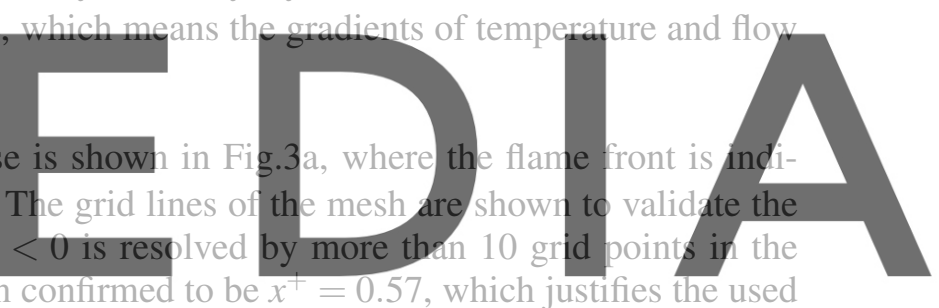
grid resolution. The straight lines in Fig. $3 a$ indi-

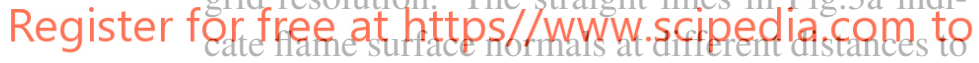

the wall (numbered from 1 to 4 ). The profiles of $\dot{r}_{\mathrm{CH}_{4}}$ along these lines are depicted in Fig.3b, with the blue curve given by the solution of a $1 \mathrm{D}$ unstretched flame. These profiles of $\dot{r}_{\mathrm{CH}_{4}}$ are shifted along the flame normal coordinate $s$, so that their peak values coincide with each other. $\left|\dot{r}_{\mathrm{CH}_{4}}\right|$ for the lines 3 and 4 is larger than $\left|\dot{r}_{\mathrm{CH}_{4}}\right|$ obtained from the $1 \mathrm{D}$ solution, which is attributable to a negative flame stretch, leading to an enhanced burning rate. The reversed effect is detected for the lines 1 and 2 closer to the wall, where the calculated $\left|\dot{r}_{\mathrm{CH}_{4}}\right|$ is smaller compared with the 1D solution. The flame experiences an intensive heat loss caused by the

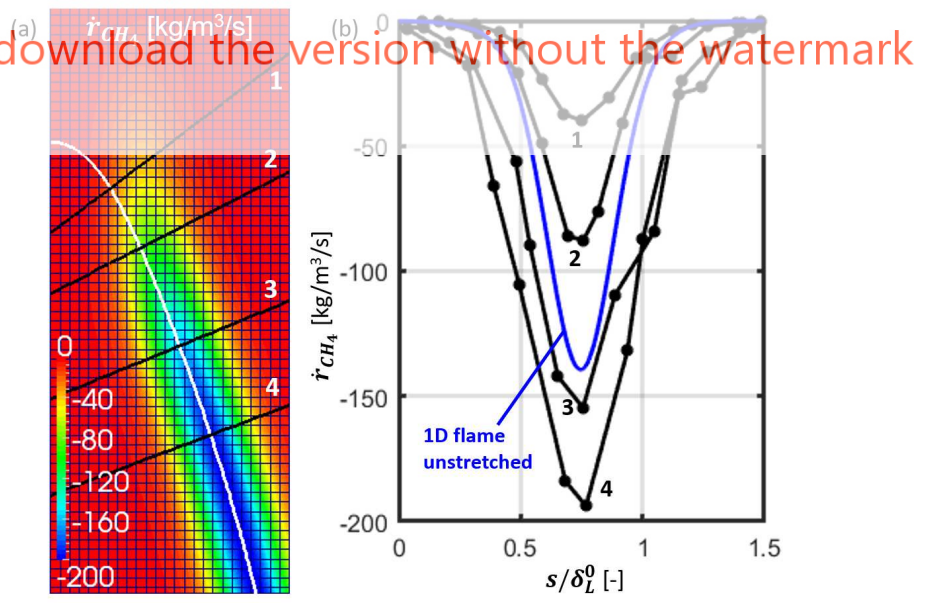
wall, which results in flame quenching or a decreased burning rate, respectively. Although not shown here, similar behavior is obtained for the other cases with $\Phi=0.8$ and 1.2. 
Figure 4 on the left shows profiles of the tangential strain component over the wall-normal distance, and in the middle for the stretch component due to curvature for the SWQ and the FF cases. $x=0$ denotes the location of the wall in case of SWQ and the symmetry plane in case of FF. Outside the flame tip or FWI zone with $x / \delta_{L}^{0}>1, K a_{s}$ and $K a_{c}$ are similar for the SWQ and FF setups, where the flame is only weakly stretched in tangential direction with $K a_{s} \approx 0.5$ and $K a_{c} \approx 0$. In the near-wall region with $x / \delta_{L}^{0}<1, K a_{s}$ reverses its sign from positive in the FF case to negative in the SWQ case. Therefore, the flame is compressed lengthwise for the SWQ case, whereas it is elongated in case of FF. The mechanism is explained in Fig.4 on the right by the contours and the streamlines of the flow velocity, where the flame fronts are shown by iso-contours of the local heat release rate. The flow velocity increases towards the flame tip, which leads to a positive tangential stretch; for the SWQ case however, the flow velocity decreases while approaching the wall, which leads to a negative tangential stretch. As the flame is negatively curved at the tip of the flame, $K a_{c}$ decreases while approaching the flame tip or the wall, as shown in Fig.4 in the middle. However, the flame is curved more strongly at the tip for the FF case compared with the SWQ case, so that the normal stretch is smaller for the FF case at the flame tip.

For the SWQ flames, 3 zones with different behaviour of flame stretch can be detected, as shown in Fig.4:

I. $K a_{s}$ decreases, whereas $K a_{c}$ or the flame curvature remains constant at $K a_{c} \approx 0$. In other words, the flame is only compressed tangentially by the flow near the wall, but not curved.

II. $K a_{s}$ continues to decrease while approaching the wall and the flame is additionally curved, leading to a negative curvature or $K a_{c}$.
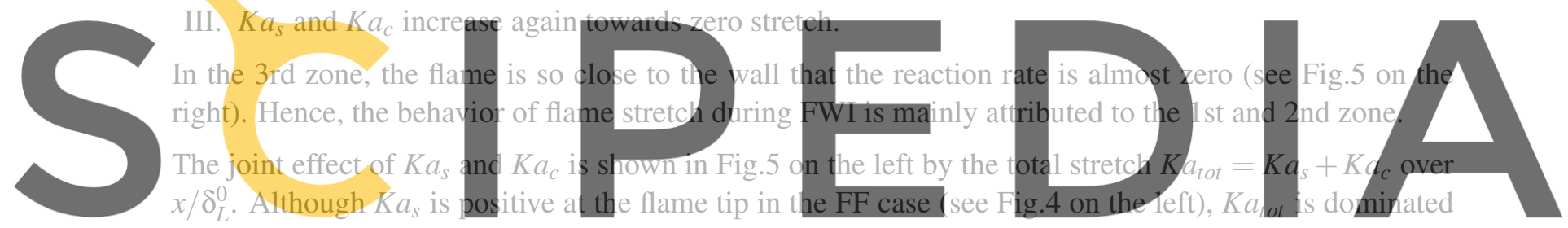

by negative values near the flame tip, indicating a stronger influence of $K a_{c}$ on $K a_{\text {tot }}$. While approaching

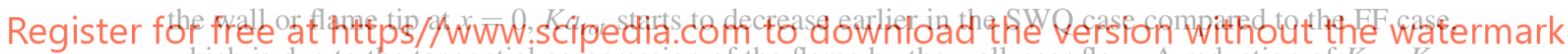

which is due to the tangential compression of the flame by the wall-near flow. A reduction of $K a_{S}, K a_{c}$

and $K a_{\text {tot }}$ with decreasing wall distance was also reported in experiments by [34] for a head-on quenching configuration with premixed methane/air flames.

Figure 5 on the right plots the normalized flame consumption speed $S_{L}$ evaluated with Eq.(3) over the wall normal distance. $S_{L}$ is largest at the flame tip with $x=0$ in case of FF and smallest with $S_{L}=0$ for the SWQ case, denoting a fully reversed response of $S_{L}$ to flame stretch. For varying $\Phi, S_{L}$ from the SWQ case (blue symbol) shows a similar distribution along the wall normal distance, where $S_{L}$ increases first with $x$ for the flame branch further away from the wall and decreases again in the region close to the wall. This leads to a local maximum of $S_{L}$ located at $x / \delta_{L}^{0} \approx 1$. The result implies that the burning rate starts to decrease or the flame starts to quench towards the wall at a distance similar to the magnitude of the laminar flame thickness. In this way, the calculated quenching distances by $d_{q} \approx \delta_{L}^{0}$ compares well with measured data by Dreizler and Böhm [2] and Häber and Suntz [6] for a SWQ configuration of rodstabilized premixed methane/air flames, although $d_{q}$ was determined in these works based on $\mathrm{OH}^{*} / \mathrm{CH}^{*}$ chemiluminescence and near-wall temperature profiles. Thus, the behavior of flame quenching can be reproduced or reflected by means of flame dynamics or flame speed, too. 


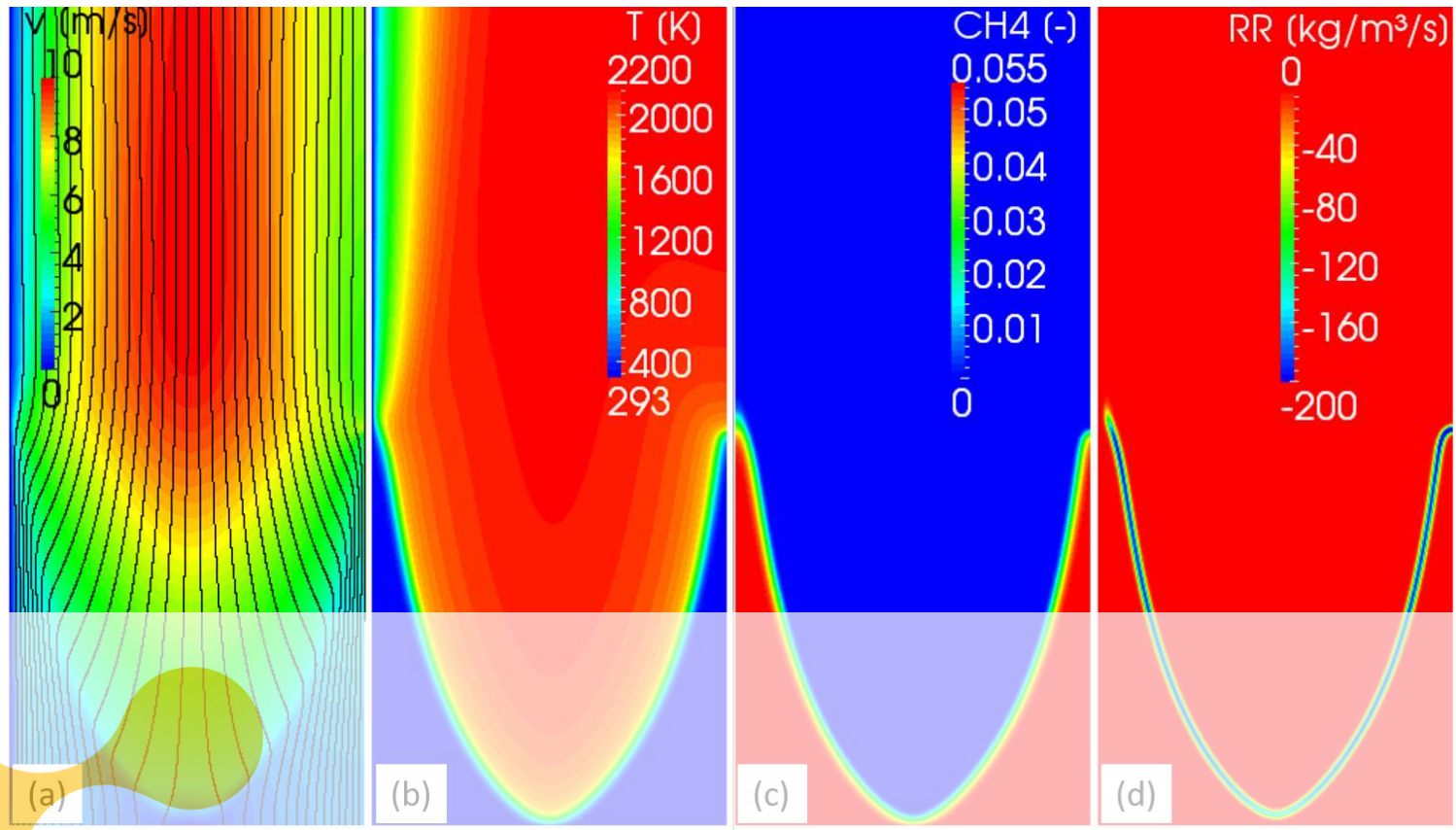

Figure 2: Calculated contours of streamwise velocity, temperature, mass fraction of $\mathrm{CH}_{4}$ and consumption rate of $\mathrm{CH}_{4}$ for the SWQ case with $\Phi=1, p_{0}=1$ bar and $T_{0}=293 \mathrm{~K}$
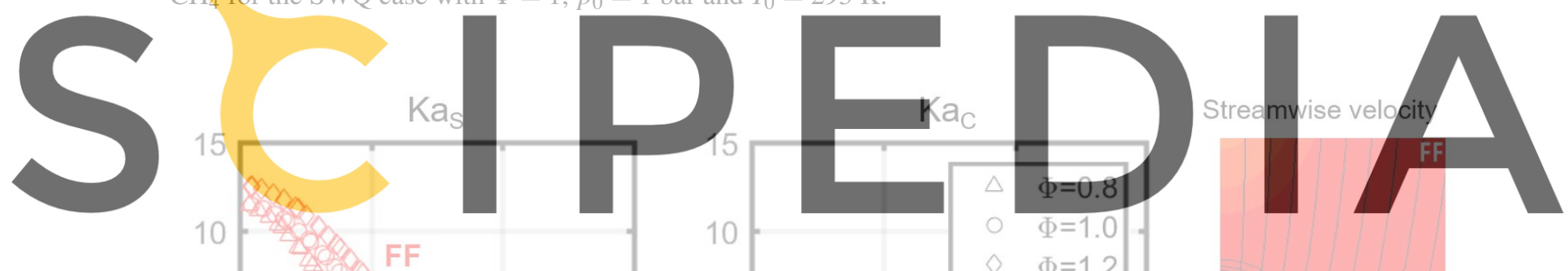

Register for free at https//www.scipedia.com to download the version without the watermark
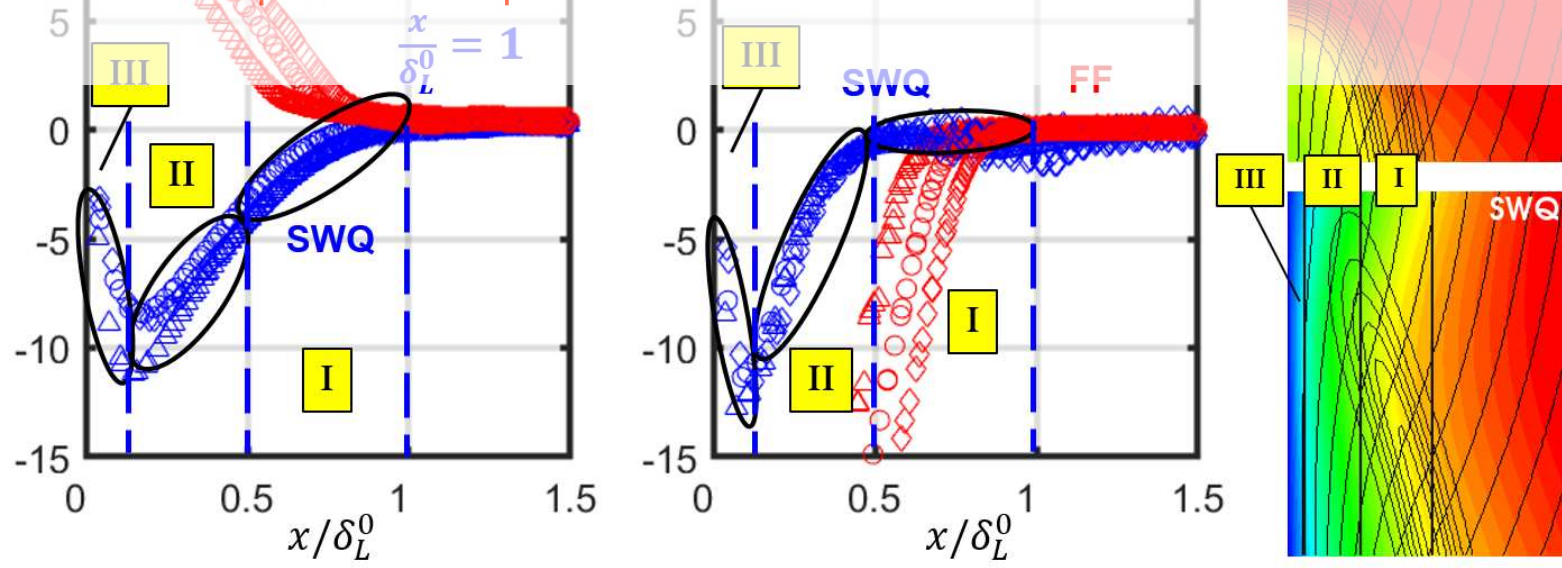

Figure 4: Comparison of tangential $K a_{s}$ (left) and normal stretch $K a_{c}$ (right) along the wall normal coordinate under SWQ (blue) and FF (red) conditions. 

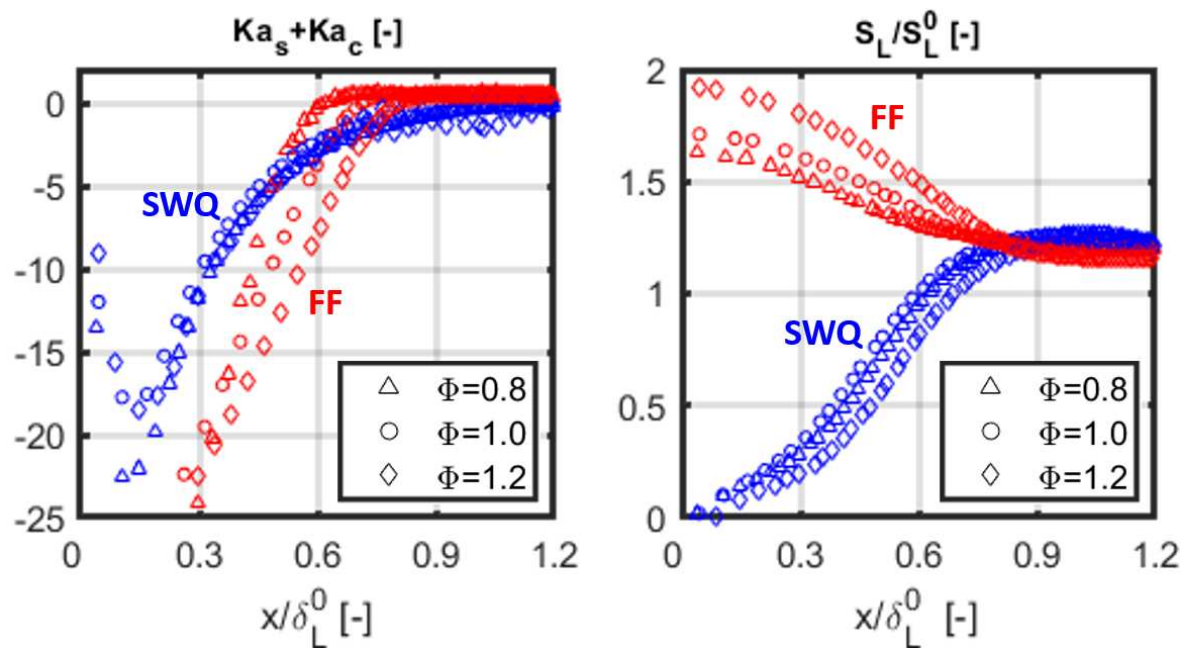

Figure 5: Comparison of $K a_{\text {tot }}$ (left) and $S_{L}$ (right) over $x$ at SWQ (blue) and FF (red) conditions.

In Fig.6, the flame speed is shown against the tangential stretch on the left, the normal stretch in the middle and the total stretch on the right, where the arrows indicate the direction towards the wall for SWQ or the flame tip for the FF case, respectively. A large number of data pairs of $S_{L}$ vs $K a$ are concentrated around $K a=0$, which represents the ordinary flame branch without influence of the flame

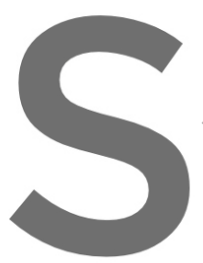
tip or FWI zone. As shown in Fig.4, $K a_{s}$ and $K a_{C}$ remain almost constant and close to $O \mathrm{fe}$
and FF cases in the range of $x / \delta_{L}^{0}>1$. The different scenarios of flame quenching close to
$x / \delta_{L}^{0}<1$ can be detected again, similar to hose for the flame stretch discussed before (sce
I. in the 1 st stage, $S_{L}$ decreases with deereasing $K \boldsymbol{d}_{s}$, while $K a_{C}$ remains almost constant
II. in the 2 nd stage, the flame is curved and $S_{L}$ decreases to 0 with both decreasing $K a$ a

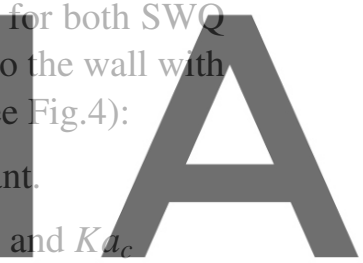

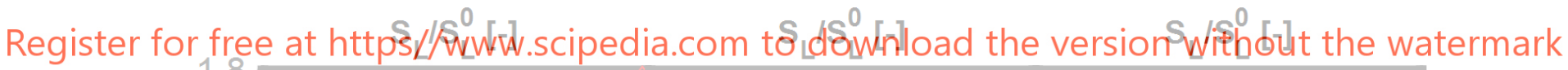

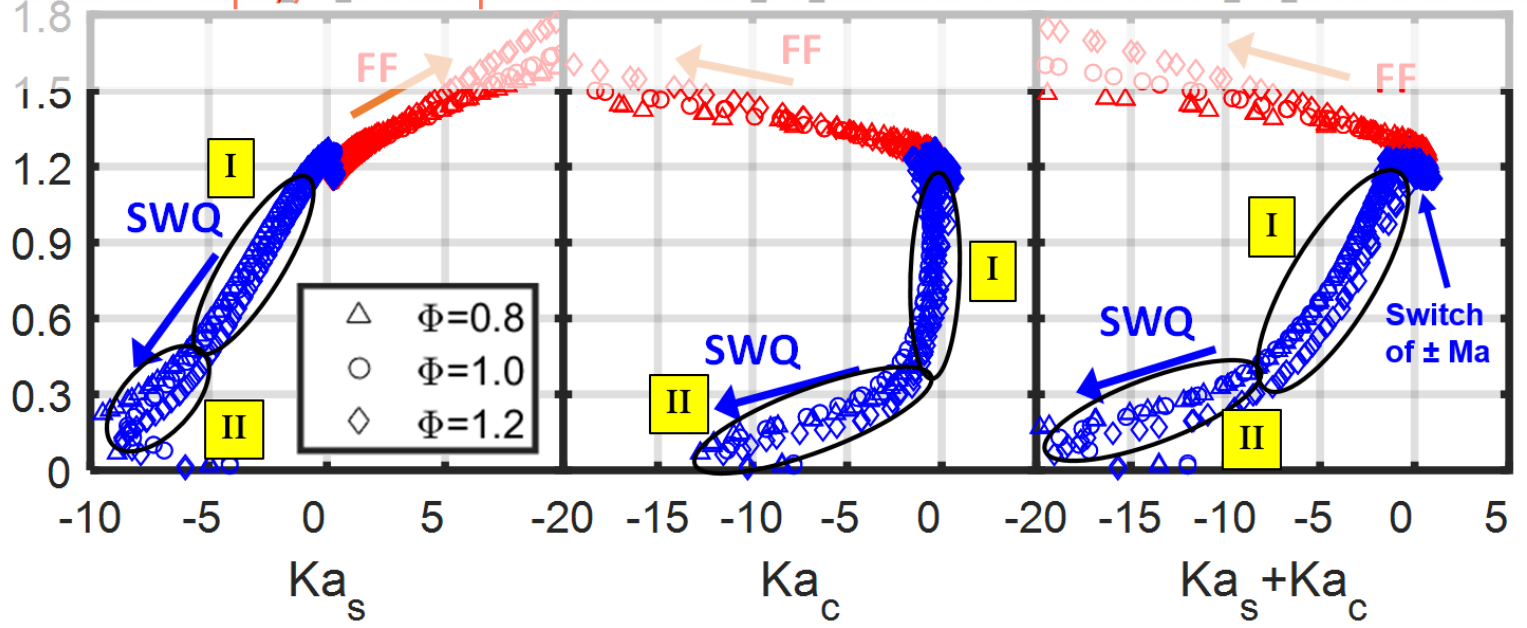

Figure 6: Correlations of flame consumption speed with flame stretch. 
In Fig.6 on the left, $S_{L}$ increases with $K a_{s}$ both for the FF and SWQ cases, revealing a negative Markstein number for the tangential strain $M a_{s}=-\frac{\mathrm{d} S_{L} / S_{L}^{0}}{\mathrm{~d} K a_{s}}$. The magnitude of $M a_{s}$ is however larger in the SWQ compared with the FF case, which can be detected from the slopes of the curves. Regarding the effect of flame curvature or normal stretch $K a_{c}$ on $S_{L}$ shown in the middle of Fig.6, $S_{L}$ decreases with decreasing $K a_{c}$ while approaching the cold wall (lower left branch) and it increases with decreasing $K a_{c}$ for the FF case (upper left branch), yielding a reversed sign of $M a_{c}$ for the SWQ and FF setups. As a consequence, $M a_{t o t}$ considering the joint effect of $K a_{s}$ and $K a_{c}$ switches its sign from positive in the FF to negative in the SWQ case, as shown in Fig.6 on the right.

For a more quantitative analysis, the Markstein number $M a$ with respect to the total stretch has been evaluated from linear fitting of the relation of $S_{L}$ vs. $K a_{S}+K a_{C}$ in Fig.7. $M a$ is positive for the FF case and negative for the SWQ case, indicating that $M a$ reverses its sign from positive to negative while approaching a cold wall, which is shown on the right of Fig.7 along with contours of the reaction rate of $\mathrm{CH}_{4}$. There, $M a$ is larger than 0 when the flame is further away from the wall, and it turns over to negative in the FWI zone. The sensitivity of $S_{L}$ to $K a_{S}, K a_{C}$ and $K a_{\text {tot }}$ are similar in magnitude for different $\Phi$ in the FWI zone, so that the FWI dynamics may be described with an almost constant $M a \approx-0.1$ for varied stoichiometries. The result reveals a close connection between flame quenching due to interaction with a cold wall and the flame dynamics in terms of flame speed and flame stretch.

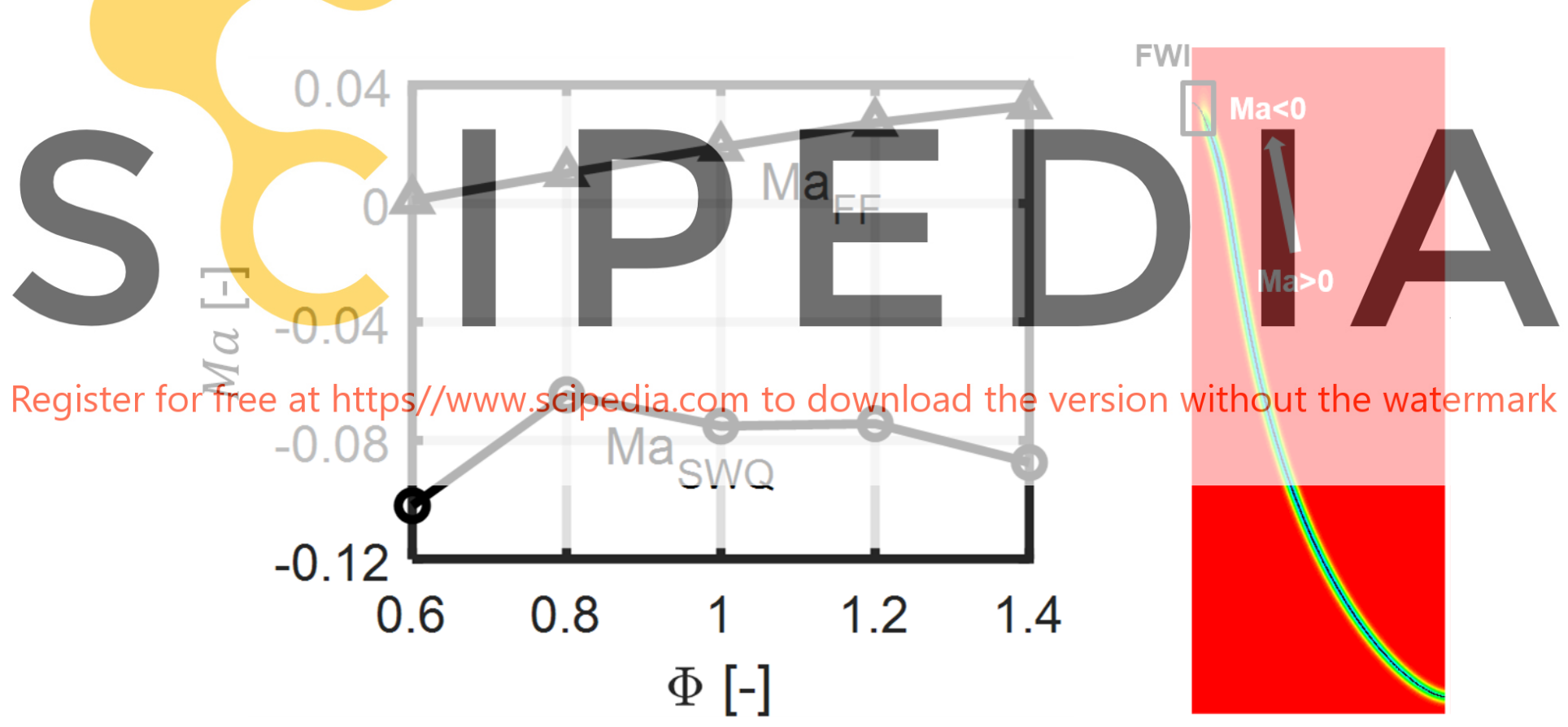

Figure 7: Calculated Markstein number for SWQ and FF flame configurations for different equivalence ratios.

\section{CONCLUSIONS}

Direct numerical simulations (DNS) have been performed to study flame dynamics of laminar premixed methane/air flames interacting with a cold wall, employing detailed calculations of the chemical reaction rates and transport coefficients. The flame's tangential and normal stretch $K a_{s}$ and $K a_{c}$, as well as the 
local flame consumption speed $S_{L}$ have been evaluated based on the DNS results. It has been shown that the flame-wall interaction (FWI) zone is dominated by negative flame stretch due to tangential compression and negative curvature of the flame. In addition, both flame stretch and flame speed decrease while approaching the wall, which results in a reversed sign of the Markstein number $M a$ from positive in the free flame branch to negative in the FWI zone. The transition location of the sign of $M a$ has been confirmed to be located at the wall distance, that is approximately equal to the unstretched laminar flame thickness. The quenching distance $d_{q}$ evaluated from this transition location of $M a$ with a maximum value of $S_{L}$ shows a quantitatively good agreement with measured data from literature.

The obtained knowledge for flame dynamics may be used for modeling the effect of FWI. For instance, for any combustion models which use the laminar or turbulent flame speed for the description of the reaction rate, a negative Markstein number can be applied in the near-wall zone to reproduce the decrease of the burning rate. Compared with common approaches for modeling FWI with tabulated chemistry or detailed kinetics, using a Markstein analogy may be more convenient and has the potential to reduce the computational cost significantly. It should be noted that the present result with inversion of the sign of the Markstein number is valid only for flames with a positive $M a$. For lean-hydrogen/air flames, $M a$ is naturally negative so that it will not reverse its sign, or the flame speed decreases due to the negative stretch in the FWI zone. Therefore, future work will study the influence of different fuels, wall temperatures, and turbulence on the flame dynamics during FWI.

\section{Acknowledgments}
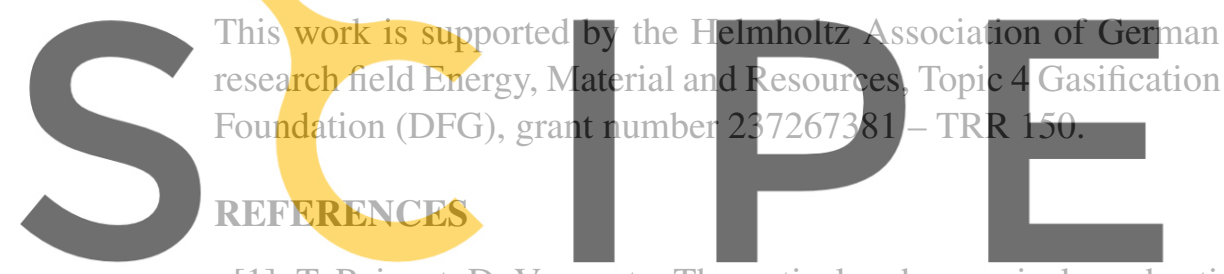

[1] T. Poinsot, D. Veynante, Theoretical and numerical combustion, RT Edwards, Inc., 2005.

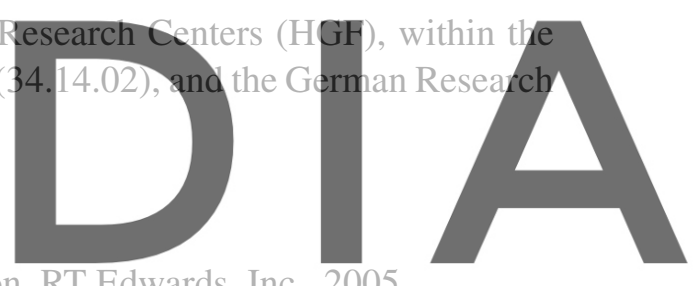

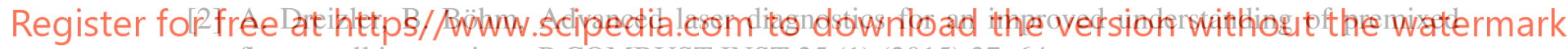
flame-wall interactions, P COMBUST INST 35 (1) (2015) 37-64.

[3] C. Jainski, M. Rißmann, B. Böhm, J. Janicka, A. Dreizler, Sidewall quenching of atmospheric laminar premixed flames studied by laser-based diagnostics, COMBUST FLAME 183 (2017) 271282.

[4] A. Heinrich, S. Ganter, G. Kuenne, C. Jainski, A. Dreizler, J. Janicka, 3d numerical simulation of a laminar experimental swq burner with tabulated chemistry, FLOW TURBUL COMBUST 100 (2) (2018) 535-559.

[5] S. Ganter, A. Heinrich, T. Meier, G. Kuenne, C. Jainski, M. C. Rißmann, A. Dreizler, J. Janicka, Numerical analysis of laminar methane-air side-wall-quenching, COMBUST FLAME 186 (2017) 299-310.

[6] T. Häber, R. Suntz, Effect of different wall materials and thermal-barrier coatings on the flame-wall interaction of laminar premixed methane and propane flames, INT J HEAT FLUID FL 69 (2018) 95-105. 
[7] C. Strassacker, V. Bykov, U. Maas, Redim reduced modeling of quenching at a cold wall including heterogeneous wall reactions, INT J HEAT FLUID FL 69 (2018) 185-193.

[8] D. Mejia, L. Selle, R. Bazile, T. Poinsot, Wall-temperature effects on flame response to acoustic oscillations, P COMBUST INST 35 (3) (2015) 3201-3208.

[9] H. Kosaka, F. Zentgraf, A. Scholtissek, L. Bischoff, T. Häber, R. Suntz, B. Albert, C. Hasse, A. Dreizler, Wall heat fluxes and co formation/oxidation during laminar and turbulent side-wall quenching of methane and dme flames, INT J HEAT FLUID FL 70 (2018) 181-192.

[10] T. Poinsot, D. C. Haworth, G. Bruneaux, Direct simulation and modeling of flame-wall interaction for premixed turbulent combustion, COMBUST FLAME 95 (1-2) (1993) 118-132.

[11] M. Saffman, Parametric studies of a side wall quench layer, Combust. Flame 55(2) (1984) 141-159.

[12] A. Gruber, R. Sankaran, E. Hawkes, J. Chen, Turbulent flame-wall interaction: a direct numerical simulation study, J. Fluid Mech. 658 (2010) 5-32.

[13] P. Popp, M. Baum, Analysis of wall heat fluxes, reaction mechanisms, and unburnt hydrocarbons during the head-on quenching of a laminar methane flame, COMBUST FLAME 108 (3) (1997) 327-348.

[14] T. Zirwes, T. Häber, F. Zhang, H. Kosaka, A. Dreizler, M. Steinhausen, C. Hasse, A. Stagni, D. Trimis, R. Suntz, H. Bockhorn, Numerical Study of Quenching Distances for Side-wall Quenching Using Detailed Diffusion and Chemistry, Flow, Turbulence and Combustion (2020). doi:10.1016/S0360-1285(98)00026-4.

[15] C. Strassacker, V. Bykov, U. Maas, Redim reduced modeling of flame quenching at a cold wallthe influence of detailed transport models and detailed mechanisms, COMBUST SCI TECHNOL 191 (2) (2019) 208-222.

[16] F. Zhang, T. Zirwes, T. Häber, H. Bockhorn, D. Trimis, R. Suntz, Near Wall Dynamics of Premixed Flames, in: Proceedings of the Combustion Institute, Vol. 38, Elsevier, 2020. doi:10.1016/j.proci.2020.06.058.

[17] T. C. Lieuwen, Unsteady combustor physics, Cambridge University Press, 2012.

[18] F. Zhang, T. Zirwes, P. Habisreuther, H. Bockhorn, Effect of unsteady stretching on the flame local dynamics, COMBUST FLAME 175 (2017) 170-179. doi:j.combustflame.2016.05.028.

[19] D. Bradley, P. Gaskell, X. Gu, Burning velocities, markstein lengths, and flame quenching for spherical methane-air flames: a computational study, COMBUST FLAME 104 (1-2) (1996) 176198.

[20] V. P. Karpov, A. N. Lipatnikov, P. Wolanski, Finding the markstein number using the measurements of expanding spherical laminar flames, COMBUST FLAME 109 (3) (1997) 436-448.

[21] T. Tahtouh, F. Halter, C. Mounaïm-Rousselle, Measurement of laminar burning speeds and markstein lengths using a novel methodology, COMBUST FLAME 156 (9) (2009) 1735-1743.

[22] F. Halter, T. Tahtouh, C. Mounaïm-Rousselle, Nonlinear effects of stretch on the flame front propagation, COMBUST FLAME 157 (10) (2010) 1825-1832.

[23] A. Bonhomme, L. Selle, T. Poinsot, Curvature and confinement effects for flame speed measure- 
ments in laminar spherical and cylindrical flames, COMBUST FLAME 160 (7) (2013) 1208-1214.

[24] F. Wu, W. Liang, Z. Chen, Y. Ju, C. K. Law, Uncertainty in stretch extrapolation of laminar flame speed from expanding spherical flames, P COMBUST INST 35 (1) (2015) 663-670.

[25] T. J. Poinsot, S. Lelef, Boundary conditions for direct simulations of compressible viscous flows, J COMPUT PHYS 101 (1) (1992) 104-129.

[26] F. Zhang, H. Bonart, T. Zirwes, P. Habisreuther, H. Bockhorn, N. Zarzalis, Direct numerical simulation of chemically reacting flows with the public domain code openfoam, in: High Performance Computing in Science and Engineering'14, Springer, 2015, pp. 221-236. doi:doi.org/10.1007/9783-319-10810-0_16.

[27] T. Zirwes, F. Zhang, J. A. Denev, P. Habisreuther, H. Bockhorn, Automated code generation for maximizing performance of detailed chemistry calculations in openfoam, in: High Performance Computing in Science and Engineering'17, Springer, 2018, pp. 189-204. doi:10.1007/978-3-31968394-2_11.

[28] T. Zirwes, F. Zhang, P. Habisreuther, M. Hansinger, H. Bockhorn, M. Pfitzner, D. Trimis, Quasi-dns dataset of a piloted flame with inhomogeneous inlet conditions, Flow, Turbulence and Combustion 104 (2019) pages997-1027. doi:10.1007/s10494-019-00081-5.

[29] T. Zirwes, F. Zhang, J. A. Denev, P. Habisreuther, H. Bockhorn, D. Trimis, Improved vectorization for efficient chemistry computations in openfoam for large scale combustion simulations, in: High Performance Computing in Science and Engineering'18, Springer, 2019, pp. 209-224. doi:10.1007/978-3-030-13325-2_13.

[30] M. Hansinger, T. Zirwes, J. Zips, M. Pfitzner, F. Zhang, P. Habisreuther, H. Bockhorn, The eulerian stochastic fields method applied to large eddy simulations of a piloted flame with inhomogeneous inlet, Flow, Turbulence and Combustion 105 (2020) 837-867. doi:10.1007/s10494-020-00159-5.

[31] T. Zirwes, F. Zhang, P. Habisreuther, M. Hansinger, H. Bockhorn, M. Pfitzner, D. Trimis, Identification of flame regimes in partially premixed combustion from a quasi-dns dataset, Flow, Turbulence and Combustion (2020) 1-32doi:10.1007/s10494-020-00228-9.

[32] T. Zirwes, F. Zhang, Y. Wang, P. Habisreuther, J. A. Denev, Z. Chen, H. Bockhorn, D. Trimis, In-situ flame particle tracking based on barycentric coordinates for studying local flame dynamics in pulsating bunsen flames, Proceedings of the Combustion Institute (2020). doi:10.1016/j.proci.2020.07.033.

[33] G. P. Smith, D. Golden, M. Frenklach, N. Moriarty, B. Eiteneer, M. Goldenberg, C. Bowman, R. Hanson, S. Song, W. Gardiner Jr, et al., Grimech 3.0 reaction mechanism, Sandia National Laboratory (2000).

[34] F. Foucher, S. Burnel, C. Mounaïm-Rousselle, M. Boukhalfa, B. Renou, M. Trinite, Flame wall interaction: effect of stretch, EXP THERM FLUID SCI 27 (4) (2003) 431-437. 